\title{
Prevalência de sintomas depressivos no idoso hospitalizado: estudo comparativo
}

\author{
Prevalence of depressive symptoms in hospitalized older adults: a comparative study
}

Prevalencia de síntomas de depresión en el anciano hospitalizado: estudio comparativo

\author{
Marcela Araujo Serra'; Isabela Maria Tavares do Nascimento"; Gilberto de Lima Guimarães"II; \\ Silmar Maria da Silva ${ }^{\prime v}$; Vania Regina Goveia ${ }^{v}$; Isabel Yovana Quispe Mendoza ${ }^{\text {I }}$
}

\begin{abstract}
RESUMO
Objetivo: comparar a prevalência dos sintomas depressivos no idoso hospitalizado, mediante uso da Escala de Depressão Geriátrica-15 e por meio da avaliação realizada pelo enfermeiro na admissão do idoso. Método: estudo descritivo de abordagem transversal em hospital público de ensino. Utilizou-se a Escala de Depressão Geriátrica e instrumento com lista de sintomas depressivos, extraídos das características definidoras dos diagnósticos de enfermagem. Resultados: a prevalência de sintomas depressivos em idosos hospitalizados foi de $47 \%$, segundo a Escala de Depressão Geriátrica, e de $25 \%$ segundo a avaliação do enfermeiro, no momento da admissão do paciente. Conclusão: a avaliação realizada pelo enfermeiro detectou baixa porcentagem dos sintomas depressivos no idoso em comparação ao instrumento específico para depressão.

Descritores: Depressão; idoso; hospitais gerais; enfermagem.
\end{abstract}

\begin{abstract}
Objective: : to compare the prevalence of depressive symptoms in hospitalized elderly using the Geriatric Depression Scale-15 and the nursing assessment of the older adult at admission. Method: this descriptive, cross-sectional study at a public teaching hospital used the Geriatric Depression Scale and an instrument listing depressive symptoms drawn from defining characteristics of nursing diagnoses. Results: the Geriatric Depression Scale returned a $47 \%$ prevalence of depressive symptoms in the hospitalized older adults, while the nursing assessment at admission found $25 \%$ prevalence. Conclusion: the nursing assessment detected a lower percentage of depressive symptoms in the older adults than the specific instrument for depression.

Descriptors: Depression; aged; hospitals, general; nursing.
\end{abstract}

\section{RESUMEN}

Objetivo: comparar la prevalencia de síntomas de depresión en el anciano hospitalizado, mediante uso de Escala de Depresión Geriátrica-15 y por medio de evaluación realizada por el enfermero en el momento de la admisión del anciano. Método: estudio descriptivo de abordaje transversal en hospital universitario. Se utilizó la Escala de Depresión Geriátrica y un instrumento con lista de síntomas de depresión, extraídos de las características definidoras de diagnósticos de enfermería. Resultados: la prevalencia de síntomas depresivos en ancianos hospitalizados fue del $47 \%$ según la Escala de Depresión Geriátrica y del $25 \%$ según evaluación del enfermero durante la admisión del paciente. Conclusión: la evaluación realizada por el enfermero detectó bajo porcentaje de síntomas de depresión en ancianos en comparación con el instrumento específico para la depresión.

Descriptores: Depresión; anciano; hospitales generales; enfermería.

\section{INTRODUÇÃO}

A prevalência de sintomas depressivos na população em geral varia de $5 \%$ a $11 \%$, sendo maior em mulheres do que em homens. Já entre os idosos, esta prevalência aumenta para 30\%. Neste grupo, a depressão encontra-se entre as doenças crônicas mais frequentes, que aumentam a possibilidade de desenvolvimento de incapacidade funcional, gastos financeiros, piora da qualidade de vida, aumento na utilização dos serviços de saúde, além de suscitar ao desfecho mais grave da doença, o suicídio ${ }^{1-4}$.

A presença de comorbidades e polifarmácia, situações comuns entre os idosos, fazem com que o diagnóstico e o tratamento adequado da depressão se tornem um processo complexo; o que pode levar a uma perda da autonomia e, consequentemente, o agravamento do quadro clínico ${ }^{5}$. Nos idosos internados, a depressão é muitas vezes ignorada pelos profissionais de saúde devido à associação dos sintomas depressivos a manifestações normais da senescência ${ }^{4}$.

'Graduanda em Enfermagem. Universidade Federal de Minas Gerais. Brasil. Email: marcellaaserra95@gmail.com.

"Enfermeira. Especialista em Saúde do Idoso. Prefeitura Municipal de Contagem, Minas Gerais. Brasil. Email: isabelamtn@gmail.com.

I'Enfermeiro. Doutor. Professor Adjunto, Universidade Federal de Minas Gerais. Brasil. Email: drgilberto.guimaraes@hotmail.com.

IVEnfermeira. Doutora. Professora Adjunta, Universidade Federal de Minas Gerais. Brasil. Email: silmarmaria@uol.com.br.

vEnfermeira. Doutora. Professora Adjunta, Universidade Federal de Minas Gerais. Brasil. Email: vaniagoveia@uol.com.br.

viEnfermeira. Doutora. Professora Adjunta, Universidade Federal de Minas Gerais. Brasil. Email: isabelyovana@gmail.com. 
Desta maneira, a avaliação de enfermagem, profissional de maior vínculo no ambiente hospitalar, deve ultrapassar os limites de uma abordagem convencional, com enfoque apenas curativo. Pois deverá ser capaz de reconhecer o sujeito em sua integralidade e identificar, precocemente, os sinais e sintomas de depressão. Tal atitude Ihe possibilitará o direcionamento para a elaboração de um plano de cuidados adequado, reduzindo as complicações clínicas de curto e longo prazo ${ }^{6}$.

Assim sendo, o uso de instrumentos que facilitam o reconhecimento da depressão deve ser estimulado. No Brasil, uma das escalas utilizadas para avaliar sintomas depressivos em populações idosas é a Escala de Depressão Geriátrica (EDG). A escala foi criada em 1983, inicialmente com 30 itens, para o rastreamento dos transtornos de humor em idosos, cujas perguntas evitam a esfera das queixas somáticas. No Brasil, esta escala foi traduzida e validada à cultura brasileira ${ }^{7}$, sendo empregadas a versão de Escala de Depressão Geriátrica-15 (EDG-15), em diferentes contextos clínicos na população idosa.

Considerando a relevância do tema, ressalta-se a importância da participação efetiva do enfermeiro na identificação dos sintomas depressivos no idoso, haja vista que estas informações podem servir para o planejamento de intervenções de enfermagem direcionadas ao paciente e família, bem como para o estabelecimento eficaz da rede de comunicação com os demais membros da equipe multidisciplinar.

Assim, este estudo teve por objetivo comparar a prevalência dos sintomas depressivos no idoso hospitalizado, mediante uso da EDG-15 e da avaliação realizada pelo enfermeiro da unidade. Os resultados deste estudo podem contribuir para melhorar a compreensão deste evento e desencadear ações que visem a identificação precoce com vistas à redução de sua ocorrência no contexto hospitalar.

\section{MÉTODO}

Trata-se de um estudo descritivo exploratório de abordagem transversal, realizado em um hospital público e de ensino, localizado em Belo Horizonte, Minas Gerais.

Para base do cálculo amostral foi considerada uma proporção esperada (p) de evento na população de $50 \%$ $(p=0,50)$, com margem de erro de $10 \%$ sobre esta estimativa $(E 0=0,10)$, o cálculo por intervalo de $95 \%$ de confiança, que resultaram em uma amostra de 96 idosos, os quais foram alocados por conveniência.

Os critérios de inclusão adotados foram: idade igual ou superior a 60 anos, internados nas unidades clínica ou cirúrgica, por no mínimo 24 horas. Adotaram-se como critérios de exclusão: pacientes com afasia, delirium e pacientes com declínio cognitivo avaliado pelo Mini Exame do Estado Mental ${ }^{8}$.

Foi elaborado, pelos pesquisadores, um instrumento de coleta de dados, composto por itens relacionados à caracterização sociodemográfica dos idosos e uma lista de sintomas depressivos, baseada nas características definidoras dos seguintes diagnósticos de enfermagem: desesperança, distúrbios da identidade pessoal, baixa autoestima crônica, baixa autoestima situacional, tristeza crônica e medo, conforme a classificação de diagnósticos de enfermagem da North American Nursing Diagnosis Association (NANDA-I) ${ }^{9}$. Foi utilizada também, a versão mais curta da escala de depressão geriátrica (EDG-15), segundo esta escala idosos com pontuação $\geq 6$, indica sintomas de depressão?.

A pesquisa aconteceu no período de maio a julho de 2016. Para iniciar a coleta de dados, realizou-se a busca ativa dos idosos nas unidades de internação clínica ou cirúrgica da instituição, a partir da lista de pacientes fornecida pelo setor de tecnologia e informática. Uma vez identificado o paciente, se apresentou a finalidade do estudo ao idoso e/ou acompanhante e, diante do aceite, foi solicitado a assinatura do termo de consentimento livre e esclarecido.

Em seguida procedeu-se a avaliação dos sintomas depressivos, pelos pesquisadores, utilizando-se o EDG-15. O prontuário eletrônico do paciente também foi consultado para a busca das características definidoras dos sintomas depressivos nos registros do enfermeiro no momento de admissão do paciente.

As variáveis categóricas foram descritas em números absolutos e percentuais. A análise foi realizada por meio do Statistical Package for the Social Sciences (SPSS), versão 19. Esta pesquisa seguiu os preceitos éticos da Resolução 466/2012 do Conselho Nacional de Saúde, que dispõe sobre as diretrizes e normas da pesquisa com seres humanos com Parecer no 1.532 .416 .

\section{RESULTADOS}

Neste estudo foram incluídos 96 pacientes. Houve predomínio do sexo feminino (54,2\%); a faixa etária predominante foi de 60 a 69 anos, (49\%); a maioria com companheiro (a) (51,1\%); com escolaridade entre um a sete anos, (75,5\%); com renda familiar entre um a três salários mínimos, $(92,7 \%)$; tendo como rede social os familiares, (82,3\%). Os resultados estão apresentados de forma detalhada na Tabela 1. 
Tabela 1 - Frequência das variáveis sociodemográficas dos idosos hospitalizados em um hospital de ensino. Belo Horizonte, MG, Brasil, 2016

\begin{tabular}{|c|c|c|}
\hline Variáveis & $\mathbf{n}$ & $f(\%)$ \\
\hline \multicolumn{3}{|l|}{ Sexo } \\
\hline Feminino & 52 & 54,0 \\
\hline Masculino & 44 & 46,0 \\
\hline \multicolumn{3}{|l|}{ Idade } \\
\hline 60 a 69 & 47 & 49,0 \\
\hline 70 a 79 & 24 & 25,0 \\
\hline 80 a mais & 25 & 26,0 \\
\hline \multicolumn{3}{|l|}{ Estado civil } \\
\hline Sem companheiro (a) & 47 & 49,0 \\
\hline Com companheiro (a) & 49 & 51,0 \\
\hline \multicolumn{3}{|l|}{ Escolaridade (anos) } \\
\hline Sem estudo & 17 & 18,0 \\
\hline 1 a 7 & 73 & 76,0 \\
\hline$\geq 8$ & 6 & 6,0 \\
\hline \multicolumn{3}{|l|}{ Rede social } \\
\hline Amigos & 17 & 17,7 \\
\hline Familiares & 79 & 82,3 \\
\hline \multicolumn{3}{|l|}{ Renda Familiar } \\
\hline$<1$ & 04 & 4,2 \\
\hline 1 a 3 & 89 & 92,7 \\
\hline$>3$ & 03 & 3,1 \\
\hline
\end{tabular}

Ao avaliar os sintomas depressivos pelo EDG-15, identificou-se que, 39\% tinham sintomas depressivos de leve a moderado, dos quais $54,1 \%$ eram do sexo feminino, e $8 \%$ apresentavam sintomas depressivos graves, distribuídos igualitariamente entre os sexos. Entretanto, 53\% dos idosos não apresentaram sintomas depressivos.

Quanto à avaliação dos sintomas depressivos, conforme a classificação de diagnósticos de enfermagem da NANDA-I e registrados pelo enfermeiro na unidade de internação, apenas $25 \%$ dos registros continham informações referentes à avaliação dos sintomas depressivos, sendo a maior proporção os idosos do sexo masculino (14,6\%). A apatia e a ansiedade foram os sintomas mais apontados pelos enfermeiros na folha de admissão do paciente, com $33 \%$ cada. Os dados estão descritos na Tabela 2.

Tabela 2 - Avaliação dos sintomas depressivos mediante Escala de Depressão Geriátrica e avaliação pelo enfermeiro, por sexo, nos idosos hospitalizados em um hospital de ensino. Belo Horizonte, MG, Brasil, 2016.

\begin{tabular}{|c|c|c|c|c|c|c|}
\hline & \multirow[b]{2}{*}{$\mathbf{n}$} & \multirow[b]{2}{*}{$f(\%)$} & \multicolumn{2}{|c|}{ Feminino } & \multicolumn{2}{|c|}{ Masculino } \\
\hline & & & $\mathbf{n}$ & $f(\%)$ & $n$ & $f(\%)$ \\
\hline \multicolumn{7}{|c|}{$\begin{array}{l}\text { Avaliação dos sintomas depressivos } \\
\text { segundo GDS }\end{array}$} \\
\hline Sem depressão GDS $<5$ & 51 & 53 & 28 & 54,9 & 23 & 45,1 \\
\hline Depressão Leve GDS 6-10 & 37 & 39 & 20 & 54,1 & 17 & 45,9 \\
\hline Depressão Grave GDS 11-15 & 08 & 08 & 04 & 50,0 & 04 & 50,0 \\
\hline \multicolumn{7}{|c|}{$\begin{array}{l}\text { Há registro de sintomas depressivos } \\
\text { identificados pelo enfermeiro? }\end{array}$} \\
\hline Sim & 24 & 25 & 10 & 10,4 & 14 & 14,6 \\
\hline Não & 72 & 75 & 42 & 43,8 & 30 & 31,2 \\
\hline \multicolumn{7}{|c|}{$\begin{array}{l}\text { Sintomas depressivos registrados pelo } \\
\text { enfermeiro }\end{array}$} \\
\hline Ansiedade & 08 & 33 & 3,0 & 5,8 & 5,0 & 11,4 \\
\hline Apatia & 08 & 33 & 2,0 & 3,8 & 6,0 & 13,6 \\
\hline Outros & 08 & 33 & 5,0 & 9,6 & 3,0 & 6,8 \\
\hline
\end{tabular}




\section{DISCUSSÃO}

Neste estudo, as características sociodemográficas dos idosos são semelhantes a estudos com idosos brasileiros que avaliaram sintomas depressivos ${ }^{4,10}$, com destaque para o predomínio do sexo feminino, com idade entre 60 a 69 anos, a baixa escolaridade, baixa renda e com companheiro; características frequentemente presentes na população brasileira e corroborados por dados do Instituto Brasileiro de Geografia e Estatística ${ }^{11}$.

A prevalência de sintomas depressivos nos idosos hospitalizados, avaliados pela EDG-15 foi de 46\%. Quando avaliada por meio dos registros do enfermeiro, identificou-se sintomas depressivos em apenas $25 \%$ dos idosos hospitalizados. A frequência de depressão em pacientes idosos é bastante variável entre os diversos cenários, mas pode chegar a aproximadamente $56 \%$ em idosos internados em hospitais gerais ${ }^{12-14}$. Assim, os resultados da presente pesquisa confirmam que a prevalência de depressão é alta em indivíduos idosos hospitalizados. Estudo refere que a depressão configura-se como objeto complexo, de difícil observação e mensuração. Pois as queixas decorrentes da presença de doenças crônicas somáticas são mais exacerbadas, tornando os sintomas depressivos menos aparentes, o que pode prejudicar seu diagnóstico ${ }^{10}$.

Identificou-se predominância do sexo feminino nos idosos com sintomas depressivos. Este dado é corroborado por estudos que mostram a prevalência de depressão no sexo feminino como sendo duas vezes mais elevada, quando comparado ao sexo masculino ${ }^{7,15,16}$. Entretanto, deve-se considerar que os homens têm dificuldade de expressar abertamente seus sentimentos, o que pode contribuir para a falsa ideia de que os transtornos depressivos acometam menos ao sexo masculino ${ }^{17}$.

Estudos mostram que a presença de sintomas depressivos está diretamente relacionada ao declínio físico, psicológico e social, o que impacta na qualidade de vida e na capacidade funcional ${ }^{18}$. Para o idoso, a situação de adoecimento, isto é, a fragilização do estado de saúde, pode ser considerada fator de risco para a presença de sintomas depressivos ${ }^{7,12,13}$.

Outro fator que pode favorecer o aparecimento de sentimentos de tristeza e desesperança é o ambiente hospitalar, uma vez que o idoso encontra-se afastado do seu domicilio e submetido a rotinas impostas pela instituição ${ }^{14}$.

O profissional de saúde tem a tendência em destacar os aspectos psicobiológicos em detrimento do psicossocial e psicoespiritual ${ }^{19}$. Historicamente, sabe-se que a construção do conhecimento dos profissionais de saúde foi baseada na relação corpo-doença, influenciado pelo modelo biomédico cartesiano e determinado pela especialização, fragmentação dos processos de trabalho e valorização da tecnologia20.

Neste estudo, pela baixa porcentagem da deteç̧ão dos sintomas depressivos, por meio da avaliação inicial do paciente pelo enfermeiro, pode-se inferir a predominância do paradigma biologicista, no qual a subjetividade humana não foi valorada. Para se libertar deste modelo é necessário o reconhecimento de que o modelo cartesiano não consegue abranger as múltiplas esferas que compõem o ser humano ${ }^{21}$.

Em contraposição, no modelo holístico todos os elementos não possuem real identidade e existência fora do seu entorno total. A saúde é considerada um grande sistema, como um fenômeno multidimensional, que envolve os aspectos físicos, psicológicos, sociais e culturais ${ }^{21}$.

Portanto, faz-se necessário refletir sobre o uso de instrumentos complementares para a avaliação do paciente, devendo assumir uma nova forma de assistir ao idoso, o que pode ser alcançado com a ampliação dos conhecimentos gerontológicos, e melhoria da prática assistencial por meio da ferramenta intelectual de trabalho do enfermeiro, o Processo de Enfermagem.

\section{CONCLUSÃO}

Os resultados do estudo mostraram alta prevalência, $46 \%$ de sintomas depressivos nos idosos hospitalizados, quando avaliados por instrumento específico para depressão. No entanto, quando avaliados no momento da admissão pelo enfermeiro apenas em $25 \%$ dos idosos foram identificados sintomas depressivos, negligenciando o diagnóstico de quase a metade dos idosos com sintomas depressivos. Este fato leva a refletir sobre a prática assistencial da enfermagem, ponderando a necessidade do uso de instrumentos específicos para avaliação dos sintomas depressivos do idoso.

O presente estudo apresenta limitações no que se refere à amostra de conveniência, não representativa da população de idosos hospitalizados, reduzindo com isto a possibilidade de encontrar resultados mais conclusivos. Quanto aos registros de enfermagem dos sintomas depressivos, neste estudo, foram considerados apenas aqueles descritos na folha de admissão. Por se tratar de pesquisa de corte transversal não foi possível conhecer a relação causa e efeito. 


\section{REFERÊNCIAS}

1. Silva MT, Galvão TF, Martins SS, Pereira MG. Prevalence of depression morbidity among Brazilian adults: a systematic review and meta-analysis. Rev. bras. psiquiatr [Internet]. 2014 [cited 2018 Mar 10]; 36(3):262-70. DOI: http://dx.doi.org/10.1590/1516-4446-2013-1294

2. Silva AR, Sgnaolin V, Nogueira EL, Loureiro F, Engroff P, Gomes I. Non-communicable chronic diseases and sociodemographic associated with symptoms of depression in elderly. J. bras. psiquiatr [Internet]. 2017 [cited 2018 Mar 05]; 66(1):45-51. DOI: http://dx.doi.org/10.1590/0047-2085000000149

3. Castro-de-Araujo LF, Barcelos-Ferreira R, Martins CB, Bottino CM. Depressive morbidity among elderly individuals who are hospitalized, reside at long-term care facilities, and are under outpatient care in Brazil: a meta-analysis. Rev. bras. psiquiatr [Internet]. 2013 [cited 2018 Mar 05]; 35(2):201-7. DOI: http://dx.doi.org/10.1590/1516-4446-2012-0905

4. Nogueira EL, Rubin LL, Giacobbo SS, Gomes I, Cataldo Neto A. Screening for depressive symptoms in older adults in the Family Health Strategy, Porto Alegre, Brazil. Rev. saúde pública [Internet]. 2014 [cited 2018 Mar 10]; 48(3):368-77. DOI: http://dx.doi.org/10.1590/S0034-8910.2014048004660

5. Chaves ECL, Paulino CF, Souza VHS, Mesquita AC, Carvalho FS, Nogueira DA. Quality of life depressive symptoms and religiosity in elderly adults: a cross-sectional study. Texto contexto enferm [Internet]. 2014 [cited 2018 Mar 25]; 23(3):648-55. DOI: http://dx.doi.org/10.1590/0104-07072014001000013

6. Ferrari AJ, Somerville AJ, Baxter AJ, Norman R, Patten SB, Vos T, et al. Global variation in the prevalence and incidence of major depressive disorder: a systematic review of the epidemiological literature. Psychol. Med [Internet]. 2013 [cited 2018 Mar 5]; 43:471-81. DOI: http://dx.doi.org/10.1017/S0033291712001511

7. Almeida OP, Almeida AS. Confiabilidade da versão brasileira da Escala de Depressão em Geriatria (GDS-15) versão reduzida. Arq. Neuropsiquiatr [Internet]. 1999 [cited 2016 Jul 15]; 57(2B): 421-6. Available from: http://www.scielo.br/pdf/anp/v57n2B/1446.pdf

8. Bertolucci PHF, Brucki SMD, Campacci SR, Juliano Y. The Mini-Mental State Examination in an outpatient population: influence of literacy. Arq. Neuropsiquiatr [Internet]. 1994 [cited 2016 Aug 12]; 52(1):1-7. DOI: http://dx.doi.org/10.1590/S0004282X1994000100001

9. NANDA Internacional. Diagnósticos de enfermagem da NANDA: definições e classificação 2012-2014. Porto Alegre: Artmed, 2012.

10. Gonzales ACT, Ignácio ZM, Jornada LK, Réus GZ, Abelaira HM, Santos MAB, et al. Depressive disorders and comorbidities among the elderly: a population-based study. Rev. bras. geriatr. Gerontol [Internet]. 2016 [cited 2018 Apr 08]; 19(1):95-103. DOI: http://dx.doi.org/10.1590/1809-9823.2016.14210

11. Instituto Brasileiro de Geografia e Estatística. Síntese de indicadores sociais: uma análise das condições de vida da população brasileira. Coordenação de População e Indicadores Sociais [Internet]. Rio de Janeiro; 2016 [cited 2018 Apr 02]. Available from: https://biblioteca.ibge.gov.br/visualizacao/livros/liv98965.pdf

12. Cohen R, Paskulin LMG, Prieb RGG. Prevalence of depressive symptoms among elderly in an emergency service. Rev. bras. geriatr. Gerontol [Internet]. 2015 [cited 2016 Aug 13]; 18(2):307-17. DOI: http://dx.doi.org/10.1590/1809-9823.2015.14052

13. Borges LJ, Benedetti TRB, Xavier AJ, D'Orsi E. Associated factors of depressive symptoms in the elderly: Epi Floripa Study. Rev. saúde pública[Internet]. 2013 [cited 2018 Aug 15]; 47(4):701-10. DOI: http://dx.doi.org/10.1590/S0034-8910.2013047003844

14. Nunes WA, Dias FA, Nascimento JS, Gomes NC, Tavares DMS. Cognition, functionality and depression indicative among elderly. Rev Rene[Internet]. 2016 [cited 2017 May 05]; 17(1):103-11. DOI: http://dx.doi.org/10.15253/2175-6783.2016000100014

15. Ferreira VMP, Silva LN, Furuya RK, Schimidt A, Rossi LA, Dantas RAS. Self-care, sense of coherence and depression in patients hospitalized for decompensated heart failure. Rev. Esc. Enferm. USP [Internet]. 2015 [cited 2018 Apr 05]; 49(3):388-94. Doi: http://dx.doi.org/10.1590/S0080-623420150000300005

16. Lopes-de-Andrés A, Jiménez-Trujillo MI, Hermández-Barrera V, Miguel-Yanes JM, Méndez-Bailón M, Perez-Farinos N, et al. Trends in the Prevalence of Depression in Hospitalized Patients with Type 2 Diabetes in Spain: Analysis of Hospital Discharge Data from 2001 to 2011. PLoS One [Internet]. 2015 [cited 2018 Apr 05]; 10(2):1-15. DOI: http://dx.doi.org/10.1371/journal.pone.0117346

17. Leão RCH, Silva VL, Moreira RS. Latent Class Analysis: a new vision of the phenomenon of depression in elderly men in the Brazilian Northeast. Rev. bras. geriatr. Gerontol [Internet]. 2017 [cited 2018 Apr 08]; 20(6):820-32. DOI: http://dx.doi.org/10.1590/1981-22562017020.160159

18. Güths JFS. Jacob MHVM, Santos AMPV, Arossi GA, Béri JU. Sociodemographic profile, family aspects, perception of health, functional capacity and depression in institutionalized elderly persons from the north coastal region of Rio Grande do Sul, Brazil. Rev. bras. geriatr. Gerontol [Internet]. 2017 [cited 2018 Apr 08]; 20(2):175-85. DOI: http://dx.doi.org/10.1590/198122562017020.160058

19. Guimarães GL, Goveia VR, Mendoza IYQ, Souza KV, Guimarães MO, Matos SS. Contribution of Horta Theory for critical of nursing diagnostics patient in hemodialysis. Rev. enferm. UFPE [Internet]. 2016 [cited 2018 Apr 10]; 10(2):554-61. Available from: https://periodicos.ufpe.br/revistas/revistaenfermagem/article/viewFile/10989/12339

20. Valença CN, Santos RCA, Medeiros SM, Guimarães J, Germano RM, Miranda FAN. Reflections on the articulacion between the homo faber and homo sapiens in nursing. Esc. Anna Nery Rev. Enferm[Internet]. 2013 [cited 2018 Apr 10]; 17(3):568-72. DOI: http://dx.doi.org/10.1590/S1414-81452013000300023

21. Teixeira E. A reflection about the holistic paradigm and holistic ad health. Rev. Esc. Enferm. USP [Internet]. 1996 [cited 2018 May 10]; 30(2):286-90. DOI: http://dx.doi.org/10.1590/S0080-62341996000200008 